\title{
XII Workshop of Physical Agents (WAF'2011)
}

\author{
Ismael García-Varea and Luis Rodríguez-Ruiz
}

\begin{abstract}
The Workshop of Physical Agents intends to be a forum for information and experience exchange in different areas regarding the concept of agent in physical environments, especially applied to the control and coordination of autonomous systems: robots, mobile robots, industrial processes or complex systems. This special issue is devoted to the selected papers presented in the WAF11 that took place in Albacete (SPAIN) from 5th to 6th of September.
\end{abstract}

Index Terms-WAF, Physical Agents.

\section{The XII EDITION OF THE WORKSHOP OF PHYSICAL AGENTS}

I INCE its first edition in 2000, the Workshop of Physical Agents has served as a meeting point for different research groups from different areas of knowledge and with a common interest: physical agents. Most of the groups that actively participate in RedAF (the Spanish Network of Physical Agents) took part in this event. RedAF is the main local sponsor organizing this Workshop. This annual event has three main objectives:

- Providing a forum for communication on research, technological innovation and technology transfer in the field of physical agents, encompassing areas such as embedded systems, artificial intelligence, mobile robotics,

- Promoting communication among researchers from different research groups related to physical agents.

- Providing a mean for active and efficient transfer of research and technological upgrading.

The XII edition was a complete success, since these goals were achieved once again. We started on September 5th, enjoying the plenary talk "Social Robots" given by Professor Carlos Balaguer from the University of Carlos III, Spain. After that, we had two working sessions on "Robots and Agents" and "Localization and Planning", consisting of four talks per session. Finally, on September 6th we attended the plenary talk "Visual Place Classification" given by Dr. Barbara Caputo from the IDIAP Research Center, Switzerland. Next, we had a working session on "Vision and Sensors", a working session on "Applications and Interaction" and, after lunch we had the demo session.

As a summary, a total of twenty papers were presented in the four working sessions. As in previous editions of the workshop, the live demos and exhibitions attracted the attention of significant media press, such as TV news and different articles in the local, regional and national newspapers, were published. We had, as well, the opportunity to interact with the mobile social robot Loky, designed by the SIMD group of

Ismael García-Varea is with the University of Castilla-La Mancha, Spain. E-mail: Ismael.Garcia@uclm.es

Luis Rodríguez-Ruiz is with the University of Castilla-La Mancha, Spain. E-mail: Luis.RRuiz@uclm.es the University of Castilla-La Mancha in conjuntion with the RoboLab group of the University of Caceres, and equiped with different elements developed by: Robotnik Automation, S.L., and IADex, S.L. These two companies also showed their last products and developments in their exhibition stands during the two days of the workshop. Also, we enjoyed the Nao Robots soccer team from the University of Rey Juan Carlos, as well as with some localization and mapping experiencies from the Robotnik patrol robots. Finally, in the closing ceremony the prize for the Best Paper of the Workshop was awarded for the first time. This prize was based on the criteria and judgement of all from the participants to the event.

In this special issue we include five papers about different topics in vision, HRI, localization and planning.

The first one, Engaging human-to-robot attention using conversational gestures and lip-synchronization, presents an algorithm for synchronizing Text-To-Speech systems with robotics mouths. The proposed approach estimates the appropriate aperture of the mouth based on the entropy of the synthetic audio stream provided by the TTS system. The authors also present the results of the opinion poll that has been conducted in order to evaluate the interaction experience.

The second one, Obstacle Avoidance in Underwater Glider Path Planning, presents a path planning scheme with low computational cost for underwater glider vehicles that allows static or dynamic obstacle avoidance, frequently demanded in coastal environments, with land areas, strong currents, shipping routes, etc. The method combines an initialization phase, inspired by a variant of the $A^{*}$ search technique and ND algorithm, with an optimization process that embraces the physical vehicle motion pattern. Consequently, the proposed method simulates a glider affected by the ocean currents, while looking for the path that optimized a given objective. According to experiments the authors carried out, this planner shows the promising results in realistic simulations, including ocean currents that vary considerably in time, and provides a superior performance over other previous approaches.

The third one, Local robot navigation based on an active visual short-term memory, proposes a dynamic visual memory to store the information gathered from a continuously moving camera onboard the robot and an attention system to decide where to look at with this mobile camera. The visual memory is a collection of relevant task-oriented objects and 3D segments, and its scope is wider than instantaneous field of view of the camera. The attention system takes into account the need reobserving objects in the visual memory, exploring new areas and testing cognitive hypotheses about object presency in the robot surroundings. The system has been programmed and validated on a real Pioneer robot that relies on the information from the visual memory for navigation tasks.

The fourth one, Multi-agent system for fast deployment of 
a guide robot in unknown environments, describes a multiagent intelligent space, which consists of intelligent cameras and autonomous guide robots. The deployment of the system does not require expertise and can be performed in a short period of time. The cameras detect situations where the robots guiding services are required, inform the robots accordingly, to posteriorly assist the robots navigation towards the goal areas, without the need of a map of the environment. An example of these situations requiring the robot guiding service could be a group of persons entering a museum. To this regard, the authors also discuss an adaptive person follower intended to be the basis of a route learning process, necessary to provide the guiding service.

The last paper of this issue, Learning in real robots from environment interaction, describes a proposal to achieve fast robot learning from its interaction with the environment. The proposal is suitable for continuous learning procedures as it tries to limit the inestability that appears every time the robot encounters a new situation it had not seen before. On the other hand, the user will not have to establish a degree of exploration (usual in reinforcement learning) and that would prevent continual learning procedures. This proposal uses an ensemble of learners able to combine dynamic programming and reinforcement learning to predict when a robot will make a mistake. This information can be used to dynamically evolve a set of control policies that determine the robot actions. This paper was the winner of the Best Paper Award of that edition of the WAF.

To conclude this introduction, we would like to thank all the authors for their excellent contributions and to extend our gratitude to all the participants of the XII Workshop of Physical Agents. We also would like to stress our gratitude to the Spanish Network of Physical Agents (http://www.redaf.es) which provides support for all the events related with physical agents in Spain. 